\title{
EL SER FOCALIZADOR EN TELENOVELAS COLOMBIANAS: EL CASO DE MEDELLÍN
}

\author{
FOCALIZING SER ('TO BE') CONSTRUCTIONS IN \\ COLOMBIAN SOAP OPERAS: THE CASE OF MEDELLÍN
}

\author{
Elena Diez del Corral Areta \\ Université de Lausanne, Faculté des Lettres \\ Elena.DiezdelCorralAreta@unil.ch
}

\begin{abstract}
Resumen:
Desde los primeros estudios sobre el ser focalizador aparecidos a finales de los ochenta, se han publicado varias investigaciones que profundizan en los aspectos formales de estas estructuras. Sin embargo, se cuenta todavía con pocos datos y se desconoce su uso en diversas zonas del dominio variacional del español. A ello pretende contribuir este trabajo, ofreciendo nuevas muestras sobre su vitalidad en una zona de Colombia poco estudiada como es Antioquia, en concreto, en Medellín. El análisis subraya, entre otras cosas, la relación estrecha que existe entre estas estructuras y las seudohendidas, su amplio uso para focalizar sintagmas preposicionales y verbos auxiliares en perífrasis verbales, y la relación inversamente proporcional que parece existir entre su empleo y el nivel socioeconómico del hablante.
\end{abstract}

Palabras clave: oraciones hendidas, telenovelas colombianas, ser focalizador, variación dialectal.

\begin{abstract}
:
Since the first studies on focalizing ser ('to be') constructions in the late eighties, some investigations that explore the formal aspects of these constructions have been published. However, there is still little data and the use of this construction in various areas of the variational Spanish domain remains unknown. The results of this work show the vitality of this construction in the scarcely studied Colombian area Antioquia, more specifically Medellin. The analysis emphasizes, among other things, the close relationship between these constructions and pseudo-clefts sentences, their widespread use to focus prepositional phrases and auxiliary verbs in periphrasis, and the inversely proportional relationship that seems to exist between their use and the socioeconomic status of the speaker.
\end{abstract}

Key words: clefts, colombian soap operas, focalizing ser ('to be') constructions, dialect variation. 


\section{Introducción}

Dentro del auge de las investigaciones sobre estructura informativa, se ha generado un creciente interés por las oraciones hendidas o escindidas ${ }^{1}$, como lo demuestran los numerosos estudios publicados a lo largo de las últimas décadas ${ }^{2}$. Aun así, existen muchas líneas abiertas de discusión sobre sus aspectos formales y funcionales, por lo que es preciso seguir analizando y contrastando las diversas construcciones en corpus multidimensionales -es decir, en corpus de textos pertenecientes a distintas tradiciones textuales (Kabatek $2006: 170$ )— que abarquen las diferentes zonas del espacio variacional del español.

En esta dirección, el presente artículo pretende ampliar nuestro conocimiento sobre un tipo de estructuras hendidas, como son las construcciones con ser focalizador ${ }^{3}$, analizándolas en una tradición discursiva poco explorada - las telenovelas - y en una variedad concreta del español: la colombiana, fundamentalmente en uno de sus dialectos (el de Medellín y sus alrededores, ubicados dentro del departamento de Antioquia).

Nuestro principal objetivo es ofrecer nuevos datos sobre este tipo de construcciones hendidas para comentar algunos de los puntos más discutidos en trabajos previos y poder así conocer mejor su uso en una región hasta ahora poco estudiada. Para ello, dedicamos un primer apartado $(§ 1)$ a la explicación de algunos aspectos controvertidos del ser focalizador, recorriendo algunas de las investigaciones más relevantes de las que disponemos. En segundo lugar (§ 2), nos detenemos en detallar el corpus que hemos confeccionado, para pasar seguidamente (§ 3) al análisis de los datos recopilados. Por último, exponemos las conclusiones de este trabajo ( $(4)$, bosquejando al mismo tiempo nuevos caminos de investigación para estudios futuros.

\footnotetext{
${ }^{1}$ Son varias las designaciones que han recibido este tipo de estructuras sintácticas en español como perífrasis de relativo (Moreno Cabrera 1983, 1999), copulativas enfáticas de relativo (NGLE 2009), construcciones ecuacionales (Alarcos 1970; Gutiérrez Ordoñez 2015; Pinuer 2005), etc. Para este trabajo empleamos el término hendida que se asemeja a la designación anglófona cleft.

2 Entre los más recientes, se encuentra el monográfico «Oraciones hendidas en el mundo hispánico: problemas estructurales y variacionales» (Diez del Corral Areta y Gutiérrez Maté 2015), que contiene trabajos de Gutiérrez Ordoñez, Feldhausen y Vanrell, Méndez Vallejo, Del Rey Quesada y Borreguero Zuloaga.

${ }^{3}$ Este término fue acuñado por Mercedes Sedano (1988) pero existen denominaciones muy variadas, previa y posteriormente a este estudio, para designar este tipo de estructuras: 'ser enfático', como las mencionó Kany (1976: 303), 'foco oracional', como las trata Toribio (2002) o 'frase de foco' dentro del sintagma verbal, como las designa Bosque (1999), entre otros.
} 


\section{El ser focalizador y las oraciones hendidas}

Para explicar las construcciones con ser focalizador se alude con frecuencia a las diferentes estructuras hendidas y a su función focalizadora ${ }^{4}$. Desde un punto de vista sintáctico, estas construcciones se caracterizan por contener tres constituyentes básicos: 1) el verbo ser (S), que funciona como elemento focalizador, 2) el elemento o constituyente focalizado (CF) y 3) una cláusula subordinada encabezada por un relativo (CS) (Dufter 2008, 1763). Según el orden sintáctico de estos elementos, obtenemos diferentes construcciones: oraciones hendidas, oraciones seudohendidas, oraciones seudohendidas inversas y construcciones con ser focalizador.

Las oraciones hendidas propiamente dichas son aquellas en las que el verbo ser precede al constituyente focalizado y a la cláusula subordinada $[(\mathrm{S})+(\mathrm{CF})+(\mathrm{CS})]$ en una frase como: Es el mal tiempo lo que me desespera. Cuando el relativo y la cláusula subordinada encabezan la oración, seguida del verbo ser y del elemento focalizado, obtenemos una oración seudohendida: Lo que me desespera es el mal tiempo $[(\mathrm{CS})+(\mathrm{S})+(\mathrm{CF})] ; \mathrm{y}$, si el elemento focalizado aparece en primer lugar, seguido del verbo ser y de la cláusula subordinada, estamos ante una oración seudohendida inversa como: El mal tiempo es lo que me desespera $[(\mathrm{CF})+(\mathrm{S})+(\mathrm{CS})]$.

Además de estas tres construcciones, existe en algunas variedades del español una cuarta en la que, si desdoblamos en dos el tercer constituyente básico — la cláusula subordinada encabezada por un relativo-, obtenemos, por un lado, un pronombre o adverbio relativo que se omite por completo, y, por otro, una cláusula incompleta, ya que a esta le falta el constituyente focalizado que puede considerarse sintáctica, semántica y pragmáticamente como un miembro de la cláusula (Sedano 1989). Obtendríamos, entonces, una oración del tipo: Me desespera es el mal tiempo $[(\mathrm{CI})+(\mathrm{S})+(\mathrm{CF})]$.

El uso del ser focalizador ha sido registrado en algunas zonas geográficas hispanohablantes como Colombia (Curnow y Travis 2003, Méndez Vallejo 2008 y 2009, Pato 2010), Venezuela (Sedano 1988, 1989 y 2003; Albor 1986), la República Dominicana (Toribio 2002), Ecuador (Toscano 1953) y Panamá (Sundheim 1922,

\footnotetext{
${ }^{4} \mathrm{Si}$ bien estas construcciones son empleadas frecuentemente para focalizar algunos elementos del enunciado, ya se ha demostrado que no todos los usos de las hendidas son estrictamente focales. Véase, por ejemplo, Dufter (2009), quien estudia las llamadas 'hendidas cohesivas' y 'hendidas inferenciales' desde un punto de vista diacrónico.
} 
Espino 1925). Asimismo, contamos con estudios sobre el español 'caribeño' (Bosque 1999 y Camacho 2006), aunque quedan todavía muchos estudios por realizar sobre el empleo y la distribución geográfica de estas construcciones dentro —e incluso fuerade estos países.

En varios de estos estudios se pueden observar dos posturas diferenciadas sobre la procedencia del ser focalizador. Por una parte, se encuentran aquellos que opinan que estas estructuras son una derivación de las oraciones seudohendidas, es decir, que existe una filiación entre ellas y, por otra parte, están los que opinan que es una estructura diferente de las seudohendidas y desligada de ellas.

La primera postura - más tradicional - considera que las construcciones con ser focalizador son una derivación de las seudohendidas, con las que solamente existe una diferencia: la omisión del relativo. Así pues, disponemos de dos estructuras muy similares, como observamos en (1) y (2):

(1) Lo que me desespera es el mal tiempo

(2) (Ø) Me desespera es el mal tiempo

El ser focalizador sería, entonces, como una reducción de las oraciones seudohendidas. Así se defiende en Albor (1986), Sedano (1990) o Toribio (2002). Guitart (2013) las denomina 'hendidas escuetas' frente a las 'hendidas plenas' y Gutiérrez Ordoñez (2015), 'ecuacionales escuetas', haciendo alusión, en ambos casos, a la diferente longitud del ser focalizador frente a otras oraciones hendidas.

Para explicar la extensión en el uso del ser focalizador, se han formulado varias hipótesis (Sedano 1989, 2003, 2010). En primer lugar, se destaca que la estructura más sencilla de las $\mathrm{SF}^{5}$ ha favorecido su uso frente a las $\mathrm{SH}$, cuya constitución es mucho más compleja. Para el emisor del enunciado es más difícil emplear SH porque el pronombre o adverbio relativo inicial tiene que concordar sintáctica y semánticamente con el $\mathrm{CF}^{6}$ (Sedano 1989: 162). Por ese motivo, las SF se emplean para focalizar los constituyentes que presentan más problemas para ser focalizados, como, por ejemplo, los sintagmas preposicionales en los que tiene que repetirse la preposición que encabeza el CF. Asimismo, para el receptor es más difícil decodificar el mensaje transmitido por las SF

\footnotetext{
${ }^{5}$ A partir de ahora utilizaremos SF cuando nos refiramos a las construcciones con ser focalizador y $\mathrm{SH}$ para hablar de las seudohendidas.

${ }^{6}$ Empleamos CF como abreviatura de: constituyente focalizado.
} 
porque no existe un relativo que permita anticipar las características sintácticosemánticas del constituyente focalizado.

Esta última idea subraya algunas de las diferencias existentes entre los CF de ambas estructuras, pues, como se recoge en Sedano (2003), al ser las SH estructuralmente complejas se emplean en contextos poco complejos - para compensar - frente a las SF que son estructuralmente sencillas y que, por tanto, son compatibles con contextos complejos. De ahí se deduce que las SH generalmente contienen un CF nominal - frente a las SF que poseen un CF generalmente preposicional, adverbial o adjetival—, y que el verbo précopula, es decir, el que precede al empleo de ser, aparece en presente en las $\mathrm{SH}-\mathrm{y}$, por tanto, ser también aparece en presente, ya que suelen concordar- mientras que en las SF se favorece el empleo de otros tiempos verbales más complejos, como el imperfecto, el perfecto simple, el condicional, el perfecto compuesto, etc. ${ }^{7}$

En cuanto a la segunda postura —más reciente-, existen ya varias investigaciones que ponen en evidencia esa filiación de las SH con las SF, como los trabajos de Curnow y Travis (2003) y Méndez Vallejo (2009, 2012, 2013), ya que las SF pueden interpretarse en muchos casos como construcciones simples a las que se añade el verbo ser. Para demostrarlo, estos autores ofrecen ejemplos de SF que no son posibles como seudohendidas y viceversa ${ }^{8}$.

De cualquier forma, lo que caracteriza a las construcciones del ser focalizador es la inexistencia de un pronombre o un adverbio relativo y que, desde el punto de vista semántico-pragmático, ofrecen el mismo valor de verdad que la oración simple correspondiente. Son construcciones de foco contrastivas - o atributivas de énfasisen las que solamente es el verbo ser el que destaca el elemento focalizado.

Además de estudios teóricos que sigan esclareciendo algunas de estas cuestiones, se requieren estudios sociolingüísticos que traten sobre la extensión y uso de estas estructuras. A estos últimos aspectos pretendemos contribuir con este trabajo, resaltando, además, algunos de los aspectos formales que acabamos de mencionar para comparar nuestros datos con los resultados de otras investigaciones. Veremos, pues,

\footnotetext{
${ }^{7}$ Retomaremos estas afirmaciones más adelante en el epígrafe 3, contrastándolas con nuestros datos.

${ }^{8}$ En el lecto colombiano, por ejemplo, es perfectamente factible una frase como No vino fue nadie, mientras que Quien no vino fue nadie es imposible. Por el contrario, una frase como Lo que hizo fue abandonarlos puede escucharse, mientras que no existe la construcción Hizo fue abandonarlos (Guitart 2013: 90). En este último ejemplo, el verbo hacer se comporta con el verbo postcópula como un pronombre en relación al nombre que sustituye y, por eso, no es posible la estructura SF.
} 
cómo este fenómeno goza de una vitalidad considerable en la actualidad, como se observa en el corpus analizado.

\section{Corpus: Escobar, el patrón del mal}

Dentro de los productos televisivos, las telenovelas ofrecen una caracterización lingüística bastante auténtica, ya que su producción es mucho más rápida que la de una película y la construcción del lenguaje depende prácticamente de un trabajo en equipo. Al igual que en los largometrajes, los guionistas escriben los discursos que se van a emitir, pero, en las telenovelas, los actores tienen mucha más flexibilidad para transformar los diálogos y decir con otras palabras aquello que han escrito los guionistas. La cotidianidad del medio televisivo y la rapidez con la que se graban, por lo general, las telenovelas, favorecen la modificación de los diálogos, pues el actor no logra memorizar literalmente todas las palabras del guion (Cisneros 2011).

Lamentablemente, no existen demasiadas investigaciones centradas en la telenovela como corpus de estudio, pues durante mucho tiempo se les ha considerado como muestras de segundo orden. Su interés científico es bastante reciente, a pesar del enorme filón que suponen para su explotación lingüística, ya que reflejan una gran riqueza dialectal al transmitir no únicamente sociolectos prestigiosos — propios del poder y de la cultura-, sino también hablas populares características de estratos bajos.

Como bien es sabido, en las últimas décadas, se ha producido un gran aumento en la realización de series y telenovelas a nivel mundial. En Colombia, una de las telenovelas más recientes es Escobar, el patrón del mal, basada en la vida del conocido narcotraficante colombiano Pablo Emilio Escobar Gaviria. Esta producción es una serie de ficción, ambientada en los años ochenta y noventa, producto de la adaptación libre de la novela La Parábola de Pablo de Alonso Salazar y de artículos de prensa y hechos históricos de público conocimiento.

Esta telenovela fue producida por Caracol Televisión entre 2009 y 2012, y se estrenó el 28 de mayo de 2012 logrando un altísimo índice de audiencia. De hecho, el primer capítulo fue el estreno más visto en la historia de la televisión colombiana. Para su realización se contó con un presupuesto muy elevado y con la participación de aproximadamente 1300 actores que grabaron en más de 500 lugares diferentes de Colombia y EEUU. Obtuvo, además, muchos premios — es la serie con más premios 
India Catalina - y fue elegida como una de las 50 producciones que cambiaron la televisión mundial.

Desde un punto de vista lingüístico, el estudio de Escobar, el patrón del mal resulta de gran interés, ya que muestra un complejo sociolingüístico en el que se privilegia el dialecto 'paisa' (característico del departamento de Antioquia y de su capital Medellín) que ha sido muy poco estudiado, sobre todo en relación con las estructuras de ser focalizador. Para este trabajo hemos analizado los 50 primeros capítulos de la serie, cada uno de una extensión que oscila entre los 30 y 60 minutos.

Del análisis de esos capítulos, hemos registrado exactamente 100 construcciones con ser focalizador, lo que nos ofrece una media aproximada de dos construcciones SF por capítulo. Los ejemplos han sido recopilados en una base de datos para su fácil manejo y estudio, teniendo en cuenta varios parámetros, como se explica en el epígrafe siguiente.

\section{Análisis}

Para el análisis de los datos hemos tenido en cuenta algunas de las variables previamente estudiadas en Sedano (2003) y Pato (2010). Destacamos cuatro: la categoría gramatical del constituyente focalizado ( $§ 3.1$ ), la función sintáctica del constituyente focalizado ( $§ 3.2$ ), el tipo de verbo (precópula) ( $§ 3.3$ ) y el tiempo, persona y número de los verbos — precópula y del ser focalizador- (§ 3.3). Además, nos interesa el vínculo que existe con las estructuras seudohendidas $(\S 3.4)$ — tal y como hemos subrayado en el epígrafe uno- y la relación entre el uso de las construcciones SF con factores extralingüísticos como el sexo, la edad y el nivel sociocultural de los hablantes $(\S 3.5)$.

\subsection{Categoría gramatical del constituyente focalizado}

Los constituyentes focalizados recopilados comprenden las siguientes categorías: sustantivos (solos) o sintagmas nominales, oraciones subordinadas sustantivas, pronombres, adjetivos, adverbios, oraciones subordinadas adverbiales, sintagmas preposicionales y gerundios o infinitivos que forman parte de perífrasis.

El tipo de categorías no difiere de las halladas por otros autores, si bien encontramos diferencias en su frecuencia de uso. Tanto Sedano (1989) como Pato (2010) resaltaban la frecuencia de adverbios, sintagmas adverbiales y subordinadas 
adverbiales como $\mathrm{CF}$, mientras que en nuestro corpus estos ocupan la sexta y séptima posición en número de ocurrencias, como se puede observar en la tabla siguiente:

\begin{tabular}{|c|c|c|}
\hline & Constituyente focalizado & Ocurrencias \\
\hline 1. & Sintagma preposicional & 31 \\
\hline 2. & Sustantivo y Sintagma nominal & 17 \\
\hline 3. & Oración subordinada sustantiva & 9 \\
\hline 4. & Pronombre & 6 \\
\hline 5. & Adjetivo y sintagma adjetival & 5 \\
\hline 6. & Adverbio y sintagma adverbial & 3 \\
\hline 7. & Oración subordinada adverbial & 10 \\
\hline 8. & Gerundio & 6 \\
\hline 9. & Infinitivo & 12 \\
\hline 10. & Conjunción (enfatizador pero ) & \\
\hline
\end{tabular}

Nuestros datos coinciden con la preferencia de estas construcciones por el sintagma preposicional como CF — como ya señalaba Sedano 1989-, pero también muestran un uso destacable para focalizar las formas no personales (o verboides) de las perífrasis verbales. En las siguientes oraciones, por ejemplo, se puede observar como el SF se emplea para focalizar el infinitivo en la perífrasis $i r+a+$ infinitivo (1), y para resaltar el gerundio en la construcción perifrástica estar+gerundio:

Perro sarnoso, deja que te agarre y te voy es a picar $(21.24$, hermano Montoa, cap. 6) ${ }^{9}$.

Conteste pues, gran pendejo, que le estoy es hablando (12.24, Pablo Escobar, cap. 6).

Asimismo, cabe destacar la categoría gramatical de conjunción (enfatizador pero) que añadimos a los CF y que no ha sido mencionada por otros estudiosos. La encontramos en ejemplos como el siguiente:

El hombre estaba era pero firme con nosotros, Pablo (22.17, Ramada,cap. 31).

\footnotetext{
${ }^{9}$ Entre paréntesis se recogen algunos datos de la construcción recopilada: se especifica el minuto exacto de la telenovela en el que se emite la construcción, la persona en concreto que la emplea y el capítulo en el que se ha registrado.
} 
Aunque el CF, en este caso, es el adjetivo firme, este viene precedido del enfatizador pero que gramaticalmente es una conjunción. Este uso, en el que el adjetivo aparece doblemente focalizado al interpolarse un elemento enfático entre el ser focalizador y el CF, merece, de cualquier manera, un estudio detallado aparte, por lo que nos limitamos únicamente a señalarlo para profundizar en él en trabajos venideros.

\subsection{Función sintáctica del constituyente focalizado}

El ser focalizador actúa como un auxiliar de predicación con una función de enlace, por lo que la función sintáctica del CF es la misma que ejercería si omitiéramos el verbo ser en la oración principal.

Los datos recopilados concuerdan, en gran medida, con los tipos de categorías gramaticales encontrados, como se puede observar en la siguiente tabla:

\begin{tabular}{|c|c|c|}
\hline & Función sintáctica & Ocurrencias \\
\hline 1. & Complemento circunstancial & 34 \\
\hline 2. & Complemento directo & 27 \\
\hline 3. & Núcleo verbal (perífrasis) & 23 \\
\hline 4. & Complemento indirecto & 0 \\
\hline 5. & Complemento de régimen & 7 \\
\hline 6. & Sujeto & 4 \\
\hline 7. & Atributo & 2 \\
\hline 8. & Predicativo & \\
\hline
\end{tabular}

La función sintáctica más habitual, con 34 ocurrencias, es la de complemento circunstancial —dado que muchos de los sintagmas preposicionales ejercen esta función-, seguida de la función de complemento directo, con 27 ocurrencias y de los 23 casos en los que se focaliza la segunda parte de una perífrasis. Más allá de estas tres funciones, los datos no son estadísticamente significativos. El foco puede ser indistintamente sujeto, complemento de régimen, atributo o predicativo.

Estos resultados contrastan en parte con lo hallado por Pato (2010), para quien el constituyente focalizado más frecuente es el que ejerce una función sintáctica de complemento directo. Estas diferencias pueden deberse probablemente a la variedad de los tipos de corpus analizados. 
3.3 Tipo de verbo (precópula), y tiempo, persona y número de los verbos (precópula y ser)

De las 100 construcciones recopiladas, se han registrado 37 tipos diferentes de verbos precópula ${ }^{10}$. En su gran mayoría son verbos de cognición, de reacción psicológica, de volición y de expresión verbal.

Según el número de ocurrencias halladas, los verbos que más favorecen el uso de ser focalizador en nuestro corpus son estar, ir, tener, venir, necesitar y querer, esto es, procesos y eventos que tienen o han tenido lugar en la realidad de los hablantes. Estos datos son similares a los hallados por otros autores, si bien Pato (2010), por ejemplo, destacaba también 'gustar' y 'dar' como verbos muy frecuentes, aunque en nuestro corpus aparece cada uno de ellos solamente en 1 ocasión.

En cuanto al SF, la forma más habitual que hemos hallado es la tercera persona del singular en presente de indicativo, es decir, es — hallada en 79 casos, lo que corresponde a un $79 \%$ del total- El segundo tiempo más frecuente es el pretérito perfecto simple (13 ocurrencias) en la tercera persona del singular (fue), seguido del pretérito imperfecto (8 casos): era.

Estos resultados difieren de lo documentado por Pato (2010), quien encuentra un $52,3 \%$ de es, un $27 \%$ de era y un $20,7 \%$ de fue, pero, sobre todo, nuestros datos contrastan con lo afirmado por Sedano (2003: 837), quien comenta que el presente de indicativo no favorece el uso de esta estructura (frente a las $\mathrm{SH}$ ) porque las SF son estructuralmente simples y, por tanto, parecen las adecuadas para transmitir información compleja frente a las SH de estructura sintáctica más compleja. En nuestro corpus, en el que se favorece el uso de los tiempos del presente, no sorprende el empleo de este tiempo verbal en el SF, pero sí resulta llamativo el alto porcentaje de ejemplos documentados, frente a lo hallado y comentado por los autores mencionados.

Generalmente el tiempo, persona y número del SF vienen condicionados por el verbo précopula. Sin embargo, encontramos diferentes combinaciones tanto en el tiempo como en la persona y número. Las personas y números del verbo precópula registrados en nuestro corpus son: 3 ps (32 casos), 1ps (26 casos), 1pp (25 casos), 3pp (15 casos) y 2 ps ( 1 caso de tú y 1 caso de vos). Estos datos contrastan también con lo

10 Los verbos en concreto son: acabar, caer, coger, conocer, considerar, contratar, dar, deber, enloquecerse, entender, entrar, estar, faltar, gastar, gustar, hablar, hacer, ir, irse, llegar, mandar, montar, necesitar, negociar, pagar, parecer, pensar, perseguir, poner, quedar, querer, ser, servir, tener, tocar, tratarse de y venir. 
encontrado por Pato (2010), quien halla que la 3ps es la más empleada (con un 41,4\%), seguida de la tercera plural (con un 36\%). La frecuente aparición de la 1ps en nuestro corpus, se puede explicar de nuevo por la telenovela analizada, ya que son habituales en ella los fragmentos en los que los interlocutores expresan su opinión, empleando la primera persona del singular.

En cuanto al tiempo del verbo precópula, normalmente corresponde con el del ser focalizador — como ya mencionábamos-, pero no siempre. Hemos encontrado casos aislados de otras formas en los que se combinan pretérito perfecto simple con presente (4), imperfecto con presente (5), condicional con presente (6), imperfecto con pretérito perfecto simple (7) y condicional con imperfecto (8):

Vino de Medellín es porque tiene algo importante que decir (6.17, Herbert, cap. 5).

Aquí se nos acaba es la fiesta (13.52, Chili, cap. 7).

Vos en vez de estar criticando, deberías es estar agradecida (10.43, Montoa, cap. 8).

Nos acabamos de quedar fue sin jefe (34.36, Pablo Escobar, cap. 2).

¡Qué va, hombre! amá, usted antes debería era apoyarme (9.02, Escobar, cap. 8).

\subsection{Reformulación de las SF en SH}

Al leer los trabajos de Sedano (1989, 2003), Toribio (2002) y otros que relacionan las seudohendidas con las estructuras de ser focalizador - mencionados en $\S$ 1 - incluimos en nuestra base de datos una rúbrica que explicitaba si era o no posible la reformulación de la SF documentada en una SH.

De los 100 ejemplos recopilados, solo 4 no pueden reformularse o, al menos, nos resultan difíciles de reformular, frente a 96 que permiten la misma estructura en una seudohendida. Los cuatro casos hallados son los siguientes:

Cada vez se hace es como más difícil conseguir recursos para las campañas políticas pero esa es la lucha (-21.25, Ortiz, cap. 7).

El primo mío y yo solamente vinimos fue de paseo (7.36, Pablo Escobar, cap. 3).

Yo voy es a pegarlo (22.37, Herbert, cap. 21).

Ustedes sí no sirven es pá nada (17.50, El coco, cap. 31).

En (9), para poder reformular la estructura en una SH, necesitaríamos cambiar la posición sintáctica del ser focalizador, incluyéndolo entre el adjetivo difícil y el infinitivo conseguir. La oración quedaría así: Lo que cada vez se hace como más difícil 
es conseguir recursos para las campañas políticas. En (10) el sintagma preposicional de paseo tendría que sustituirse por una subordinada final como para pasear, modificando así la oración: Para lo que el primo mío y yo solamente vinimos fue para pasear. En (11) la reformulación de la SF en SH resulta también imposible (*a lo que voy es a pegarlo), al igual que en (12), en la que la negación impide la reformulación (*Para lo que no sirven es para nada).

Estos resultados, de cualquier manera, demuestran que el uso del ser focalizador todavía es mayor en estructuras que permiten el añadir la cláusula de una subordinada relativa. La filiación entre las SH y las SF es, por tanto, evidente, aunque se hallen algunos ejemplos de SF que no permitan la reformulación en $\mathrm{SH}$.

\subsection{Factores extralingüísticos}

En uno de los pioneros estudios sociolingüísticos sobre el ser focalizador (Sedano 1990) se destaca cómo el uso del SF es inversamente proporcional al nivel socioeconómico del informante. Sedano (1990) documenta un uso de un $10 \%$ en hablantes de un nivel socioeconómico alto, un $16 \%$ en un nivel socioeconómico medio y un $22 \%$ en un nivel socioeconómico bajo. Esta autora constata, además, que la edad no influye en el grupo bajo, pero sí en el medio y en el alto, siendo los hombres de nivel bajo y medio, y las mujeres de la generación joven los que más favorecen el uso de esta estructura.

En nuestro corpus es difícil estudiar estos factores de manera proporcionada porque no son equitativas las intervenciones de personas de diferentes clases sociales, sexos o edades. Los protagonistas de la novela son hombres de una clase social baja, por lo que las intervenciones de personas pertenecientes a una clase social alta son más escasas. Las mujeres, además, no participan tanto en las conversaciones y los personajes, en general, son todos de una edad adulta.

Nuestros resultados, por tanto, presentan ciertos límites, aunque no dejan de ser significativos para el tipo de corpus analizado. De las 100 muestras de SF recopiladas, 93 son enunciadas por narcotraficantes y sicarios del cartel de Medellín. De esas 93, además, sobresale la cantidad de casos emitidos por Pablo Escobar: en total, 40.

De políticos y periodistas hemos registrado únicamente 2 casos respectivamente. En cuanto a las mujeres, se han recopilado solamente 3 ejemplos: 2 emitidos por mujeres pertenecientes a una clase social baja (1 por la mujer de Pablo Escobar y 1 por 
la hermana prostituta de uno de sus sicarios) y 1 por una mujer perteneciente a una clase social media: la mujer del Coronel Jiménez.

Con estos datos, no podemos ofrecer un estudio sociolingüístico riguroso que tenga en cuenta la edad, el sexo y el nivel sociocultural de los hablantes, pero sí comentar que estos resultados parecen estar relacionados con lo afirmado por Sedano (1990) o, al menos, no lo desmienten.

De cualquier manera, además, cabe subrayar que los enunciados analizados son emitidos por actores que están representando a unos personajes determinados y, por tanto, no a personas reales. Por ello, el corpus no nos permite afirmar conductas lingüísticas específicas en términos de frecuencia de uso que constituyan el perfil de una clase social determinada.

\section{Conclusión}

Retomando los resultados del análisis de este estudio, cabe destacar que los sintagmas preposicionales siguen siendo el tipo de categoría gramatical más empleada como constituyente focalizado, tal y como han encontrado otros autores. En nuestro corpus destaca el uso del SF entre los verbos auxiliares y las formas verbales no personales que constituyen las perífrasis, además de algunos ejemplos en los que el constituyente focalizado va encabezado por la conjunción pero con un valor enfático.

Por otro lado, y si comparamos los datos con lo señalado por Sedano (2003), al contrastar los tipos de CF que diferencian las SH de las SF, llama la atención que el constituyente focalizado en nuestro corpus es también habitualmente nominal. De hecho, es el tercer tipo de CF más frecuente después de los sintagmas preposicionales y los núcleos verbales de las perífrasis, lo que destaca frente a otros estudios que atestiguaban sobre todo adverbios $\mathrm{u}$ oraciones subordinadas adverbiales. Estos resultados pueden deberse a la clara extensión y difusión de esta estructura en la actualidad que focaliza ya cualquier tipo de elemento, incluyendo los constituyentes focalizados más habituales en las $\mathrm{SH}$.

En cuanto a la variable del tiempo del verbo precópula, sobresale el hecho de que el verbo aparece principalmente en presente de indicativo, a pesar de que Sedano 1990 (en la tabla) indicaba que este tiempo era el primordial para las SH, mientras que en las SF se favorecían otros tiempos verbales. Estos resultados pueden deberse a la particularidad de nuestro corpus en el que se emplean frecuentemente diálogos referidos al presente y muchas veces en primera persona del singular, lo que favorece la 
diversidad de datos frente a lo hallado en otros estudios. Por otro lado, estas diferencias pueden deberse a un posible cambio lingüístico de los trabajos y corpus de los años 90 , frente a corpus actuales como el analizado en este trabajo.

Asimismo, hay que tener en cuenta las posibles diferencias dialectales, ya que nuestro corpus se limita a una zona poco estudiada, cuyos datos deben contrastarse con otros corpus de la misma área lingüística. Para estudios futuros, además, estos datos deberían compararse con corpus similares de otras regiones representativas de variedades colombianas como, por ejemplo, los obtenidos de alguna telenovela que se ubique en la zona de la costa o en otras zonas del interior.

Así pues, quedan pendientes muchos estudios que sigan investigando las propiedades no solo sintácticas de estas estructuras, sino semánticas, pragmáticas e incluso prosódicas, pues, una vez demostrada la vitalidad que posee este tipo de construcciones en la actualidad, hay que seguir analizándola para poder alcanzar un mayor conocimiento sobre sus propiedades y su difusión en el dominio variacional del español.

\section{Bibliografía}

Alarcos, Emilio. Estudios de sintaxis funcional del español. Madrid: Gredos, 1970. Impreso.

Albor, Hugo R. "Uso e interpretación de ser en construcciones galicadas y en Él necesita es descansar". Thesaurus 41(1986): 173-186. Impreso.

Bosque, Ignacio. "On focus vs. wh-movement: The case of Caribbean Spanish". Sophia Lingüística 44-45 (1999): 1-32. Impreso.

Camacho, José: "In Situ focus in Caribbean Spanish: Towards a unified account of focus". Selected Proceedings of the 9th Hispanic Linguistic Symposium. Eds. Nuria Sagarra y A. Jacqueline Toribio. Somerville: Cascadilla Proceedings, 2006: 13-23. Impreso.

Cisneros Estupiñán, Mireya: "Lenguaje y sociedad en la telenovela colombiana del XXI". Variación del español en los medios. Ed. Raúl Ávila. México D. F.: El Colegio de México, 2011: 247-266. Impreso.

Curnow, Timothy J. y Travis Catherine E. "The emphatic es construction of Colombian Spanish". Proceedings of the 2003 Conference of the Australian Linguistic Society. Ed. Christo Moskovsky. Newcastle: ALS, 2003: 1-11. Impreso.

Diez del Corral Areta, Elena y Gutiérrez Maté, Miguel. (Coord.). "Sección temática Oraciones hendidas en el mundo hispánico: problemas estructurales y variacionales". Revista Internacional de Lingüística Iberoamericana, 26 (2015):7-13. Impreso.

Dufter, Andreas: "Evolución pragmática de las oraciones hendidas en español: el papel de los usos no focalizadores". Actas del VII Congreso Internacional de la Asociación de Historia de la Lengua Española. Eds. C. Company Company y J. Moreno de Alba. Madrid: Arco Libros, 2008. 1763-1779. Impreso. 
--------. "Clefting and Discourse Organization: Comparing Germanic and Romance". Focus and Background in Romance languages. Eds. Andreas Dufter y Daniel Jacob. Amsterdam: John Benjamins, 2009: 83-121. Impreso.

Espino, Lisandro. Ensayo de crítica gramatical. Panamá: Editorial La Moderna, 1925.

Guitart, Jorge M. "Del uso de las oraciones hendidas en el español actual". Revista Internacional d'Humanitats 27 (2013): 89-104. Impreso.

Gutiérrez Ordoñez, Salvador. "La familia de las ecuacionales". Oraciones hendidas en el mundo hispánico: problemas estructurales y variacionales. Eds. Elena Diez del Corral Areta y Miguel Gutiérrez Maté. Revista Internacional de Lingüística Iberoamericana, 26.26 (2015): 15-37. Impreso.

Kabatek, Johannes. "Tradiciones discursivas y cambio lingüístico". Eds. Konstanze Jungbluth, Dorothee Kaiser y Célia Lopes. Sincronía y diacronía de tradiciones discursivas en Latinoamérica. Madrid/Frankfurt am Main: Iberoamericana/Vervuert, 2006: 151-173. Impreso.

Kany, Charles. Sintaxis Hispanoamericana. Madrid: Gredos, 1976. Impreso.

Méndez Vallejo, Dunia Catalina. "Some Syntactic Considerations Regarding the Focalizing Ser ('to be') Construction in Colombian Spanish". Eds. Michael Grosvald y Dionne Soares. Proceedings of the 38th Western. Conference on Linguistics, 2008: 208-219. Impreso.

Focalizing SER ('to be') in Colombian Spanish. Indiana University, Bloomington, Tesis doctoral, 2009. Impreso.

."On the syntax of the Focalizing ser ('to be') structure in the Spanish of Bucaramanga”. Eds. R. File-Muriel y R. Orozco. Colombian varieties of Spanish. Madrid/Frankfurt: Iberoamericana/Vervuert, 2012. Impreso.

------. Focalizing ser ('to be') in Colombian Spanish. An empirical study of a diallectally-marked syntactic phenomenon in Spanish. Lambert Academic Publishing, 2013. Impreso.

Moreno Cabrera, Juan Carlos. "Las perífrasis de relativo". F. Lázaro Carreter, Serta Philologica. I. Madrid: Castalia, 1983: 455-467. Impreso.

-."Las funciones informativas: las perífrasis de relativo y otras construcciones perifrásticas". Eds. Ignacio Bosque y Violeta Demonte. Gramática descriptiva del español actual. Tomo III. Madrid: Espasa, 1999: 4245-4302. Impreso.

Nueva Gramática de la Lengua Española, Real Academia Española y Asociación de academias de la lengua española. Nueva Gramática de la Lengua Española. Madrid: Espasa Calpe, 2009. Impreso.

Pato, Enrique. "El verbo ser focalizador en el español de Colombia". Español Actual, 93 (2010): 151-172. Impreso.

Pinuer, Claudio. "Relieve sintáctico en el español escrito de Chile: Las construcciones ecuacionales y ecuandicionales". Signos, 38.57 (2005): 75-88.

Sedano, Mercedes. "Yo vivo es en Caracas: un cambio sintáctico". Eds. R. Hammond y M. Resnick. Studies in Caribbean Spanish Dialectology. Washington: Georgetown University Press, 1988: 115-123. Impreso.

."Un análisis comparativo de las cláusulas seudohendidas y de las cláusulas con verbo ser focalizador en el habla de Caracas". Vol. 2. Actas del VII Congreso de la Asociación de Lingüística y Filología de América Latina. Santo Domingo: ALFAL, 1989: 157-172. Impreso.

"Más sobre las seudohendidas y construcciones con verbo ser focalizador

en el habla de Caracas". Vol. 2. Eds. Francisco Moreno Fernández et al. Lengua, variación y contexto: estudios dedicados a Humberto López Morales. España: Arco, 2003: 823-847. Impreso. 
-----. "El verbo ser en las oraciones seudohendidas y con verbo ser focalizador". Nueva Revista de Filología Hispánica, 58. 1 (2010): 39-58. Impreso.

Sundheim, Adolfo. Vocabulario costeño o lexicografía de la región septentrional de la república de Colombia. París: Librería Cervantes, 1922. Impreso.

Toribio, Almeida Jacqueline. "Focus on Clefts in Dominican Spanish". James F. Lee et al. (eds.), Structure, Meaning and Acquisition in Spanish. Somerville, M.A: Cascadilla Press, 2002. Impreso.

Toscano, Humberto. El español hablado en el Ecuador. Madrid: CSIC, 1953. Impreso. 